International Journal of Pure and Applied Mathematics

Volume 105 No. 2 2015, 153-162

ISSN: 1311-8080 (printed version); ISSN: 1314-3395 (on-line version)

url: http://www.ijpam.eu

doi: http://dx.doi.org/10.12732/ijpam.v105i2.3

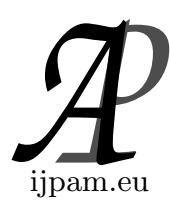

\title{
OPERATIONS ON $S$-GRAPHS
}

\author{
M. Rajkumar ${ }^{1}$, S. Jeyalakshmi ${ }^{2}$, M. Chandramouleeswaran ${ }^{3}$ § \\ ${ }^{1,3}$ Saiva Bhanu Kshatriya College \\ Aruppukottai, 626101, Tamilnadu, INDIA \\ ${ }^{2}$ Devanga Arts College \\ Aruppukottai, 626101, Tamilnadu, INDIA
}

\begin{abstract}
In our earlier paper (see [4]), we have introduced the notion of semiring-valued graphs and (see [3]) the notion of regularity on $S$-graphs. In this paper, we introduce and study some operations such as union and sum of two $S$-graphs.
\end{abstract}

AMS Subject Classification: 05C25, 16Y60

Key Words: $S$-graphs, regular $S$-grpahs, operations on $S$-graphs

\section{Introduction}

Eventhough the concept of semiring was first introduced by H.S.Vandiver [5] in 1934, the developments of the theory in semirings and ordered semirings have been taking place since 1950. Jonathan S.Golan [2] has introduced the notion of $S$-graph where he considers a function $g: V \times V \rightarrow S$ such that $g\left(v_{1}, v_{2}\right) \neq 0$. But nothing more has been dealt. This motivated us to study graphs whose vertices and edges are assigned values from the semiring $S$ [4]. Golan considers the $S$-graph by assigning values to edges only. However we assign values to every vertex of the graph and the weights of an edge is assigned in relation to the weights of the vertices incident with the edges. Since every semiring

Received: August 5, 2015

(C) 2015 Academic Publications, Ltd.

$\S$ Correspondence author url: www.acadpubl.eu 
possesses a canonical pre-order, for any edge $e=\left(v_{i}, v_{j}\right)$, we can assign the weight of $e$ as the minimum weights of $v_{i}$ and $v_{j}$. Such a graph we called a $S$-graph. In our paper [3], we studied the notion of regularity on $S$-graphs. In this paper, we introduce and study the notion of operations such as union and sum of two $S$-graphs.

\section{Preliminaries}

In this section, we recall the basic definitions on operations on crisp graphs, such as union and sum of two graphs. For graph theoretical concepts, we refer [1].

Definition 2.1. Let $G_{1}=\left(V_{1}, E_{1}\right)$ and $G_{2}=\left(V_{2}, E_{2}\right)$ be two graphs with $V_{1} \cap V_{2}=\phi$. We define the Union $G_{1} \cup G_{2}=(V, E)$ where $V=V_{1} \cup V_{2}$ and $E=E_{1} \cup E_{2}$.

Definition 2.2. The sum $G_{1}+G_{2}$ as $G_{1} \cup G_{2}$ together with all the lines joining points of $V_{1}$ to points of $V_{2}$.

Definition 2.3. A semiring $(S,+, \cdot)$ is an algebraic system with a nonempty set $S$ together with two binary operations + and $\cdot$ such that

1. $(S,+, 0)$ is a monoid.

2. $(S, \cdot)$ is a semigroup.

3. For all $a, b, c \in S, a \cdot(b+c)=a \cdot b+a \cdot c$ and $(a+b) \cdot c=a \cdot c+b \cdot c$.

4. $0 \cdot x=x \cdot 0=0 \forall x \in S$.

Definition 2.4. Let $S$ be a semiring. $\preceq$ is said to be a Canonical Pre-order if for $a, b \in S, a \preceq b$ if and only if there exists $c \in S$ such that $a+c=b$.

Definition 2.5. [4] Let $G=(V, E \subset V \times V)$ be the underlying graph with both $V, E \neq \phi$. For any semiring $(S,+, \cdot)$, a Semiring-valued graph (or an $S$ valued graph) $G^{S}$ is defined to be the graph $G^{S}=(V, E, \sigma, \psi)$ where $\sigma: V \rightarrow S$ and $\psi: E \rightarrow S$ is defined to be

$$
\psi(x, y)=\left\{\begin{array}{cc}
\min \{\sigma(x), \sigma(y)\} & \text { if } \sigma(x) \preceq \sigma(y) \text { or } \sigma(y) \preceq \sigma(x) \\
0 & \text { otherwise }
\end{array}\right.
$$

for every unordered pair $(x, y)$ of $E \subset V \times V$. We call $\sigma$, a $S$-vertex set and $\psi$, a $S$-edge set of $S$-valued graph $G^{S}$.

Henceforth we call a $S$-valued graph simply as a $S$-graph. 
Definition 2.6. [4] If $\sigma(x)=a, \forall x \in V$ and some $a \in S$ then the corresponding $S$-graph $G^{S}$ is called a vertex regular $S$-graph (or simply vertex regular).

Definition 2.7. [4] An $S$-graph $G^{S}$ is said to be an edge regular $S$-graph (or simply edge regular) if $\psi(x, y)=a$ for every $(x, y) \in E$ and some $a \in S$.

Definition 2.8. [4] An $S$-graph $G^{s}$ is said to be $S$-regular if it is both vertex regular and edge regular .

Definition 2.9. [3] Let $G^{S}$ be an $S$-graph corresponding to an underlying graph $G$. $G^{S}$ is said to be $(a, k)$-regular $S$-graph if the following conditions are true.

1. The crisp graph $G$ is $k$-regular.

2. $\sigma(v)=a$ for every vertex $v$ in $G$.

\section{Operations on $S$-Graph}

In this section, we define the notion of union and sum of two $S$-graphs and prove some simple results.

Definition 3.1. Let $G_{1}^{S}=\left(V_{1}, E_{1}, \sigma_{1}, \psi_{1}\right)$ and $G_{2}^{S}=\left(V_{2}, E_{2}, \sigma_{2}, \psi_{2}\right)$ be two $S$-graphs corresponding to the underlyings graphs $G_{1}=\left(V_{1}, E_{1}\right)$ and $G_{2}=$ $\left(V_{2}, E_{2}\right)$ with $V_{1} \cap V_{2}=\phi$ respectively. Then their union, denoted by $G_{1}^{S} \cup G_{2}^{S}$, is defined as $G_{1}^{S} \cup G_{2}^{S}=(V, E, \sigma, \psi)$ where $V=V_{1} \cup V_{2} ; E=E_{1} \cup E_{2}$ and for $v \in V$, $\sigma(v)=\left\{\begin{array}{ll}\sigma_{1}(v) & \text { if } v \in V_{1} \\ \sigma_{2}(v) & \text { if } v \in V_{2}\end{array} ;\right.$

For $\left(v_{i}, v_{j}\right) \in E=E_{1} \cup E_{2}, \psi\left(v_{i}, v_{j}\right)= \begin{cases}\psi_{1}\left(v_{i}, v_{j}\right) & \text { if }\left(v_{i}, v_{j}\right) \in E_{1} \\ \psi_{2}\left(v_{i}, v_{j}\right) & \text { if }\left(v_{i}, v_{j}\right) \in E_{2}\end{cases}$

Remark 3.2. Clearly $G_{1}^{S} \cup G_{2}^{S}$ is an $S$-graph. In general, $G_{1}^{S} \cup G_{2}^{S} \neq$ $\left(G_{1} \cup G_{2}\right)^{S}$.

Example 3.3. Let $(S=\{0, a, b\},+, \cdot)$ be a semiring with the following Cayley Tables:

\begin{tabular}{|c|c|c|c|}
\hline+ & 0 & $a$ & $b$ \\
\hline 0 & 0 & $a$ & $b$ \\
\hline$a$ & $a$ & $o$ & $b$ \\
\hline$b$ & $b$ & $b$ & $b$ \\
\hline
\end{tabular}

\begin{tabular}{|c|c|c|c|}
\hline$\cdot$ & 0 & $a$ & $b$ \\
\hline 0 & 0 & 0 & 0 \\
\hline$a$ & 0 & 0 & 0 \\
\hline$b$ & 0 & 0 & $b$ \\
\hline
\end{tabular}


Clearly $\preceq$ is a canonical pre-order in $S$, where

$$
0 \preceq 0,0 \preceq a, 0 \preceq b, a \preceq a, a \preceq 0, a \preceq b, b \preceq b .
$$

Let $G_{1}$ and $G_{2}$ be two crisp graphs given by :
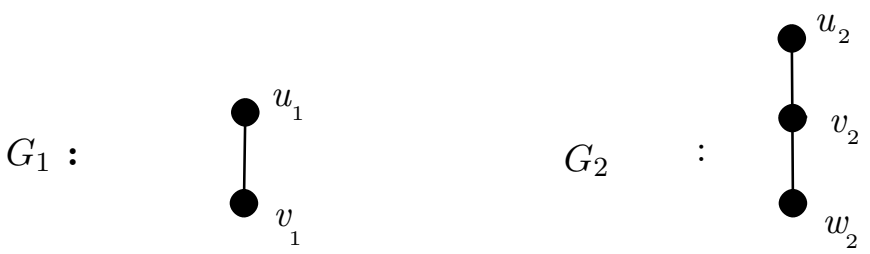

Here $V_{1}=\left\{u_{1}, v_{1}\right\}$ and $V_{2}=\left\{u_{2}, v_{2}, w_{2}\right\}$

$E_{1}=\left\{\left(u_{1}, v_{1}\right)\right\}$ and $E_{2}=\left\{\left(u_{2}, v_{2}\right),\left(v_{2}, w_{2}\right)\right\}$

$\sigma_{1}: V_{1} \rightarrow S$ is defined by $\sigma_{1}\left(u_{1}\right)=\sigma_{1}\left(v_{1}\right)=a$ and $\psi_{1}\left(u_{1}, v_{1}\right)=a$.

And $\sigma_{2}: V_{2} \rightarrow S$ is defined by $\sigma_{2}\left(u_{2}\right)=\sigma_{2}\left(v_{2}\right)=a$ and $\sigma_{2}\left(w_{2}\right)=b$.

Then $\psi_{2}\left(u_{2}, v_{2}\right)=\psi_{2}\left(v_{2}, w_{2}\right)=a$.

Therefore the union of the $S$-graphs $G_{1}^{S}$ and $G_{2}^{S}$ is the $S$-graph $G_{1}^{S} \cup G_{2}^{S}$ :

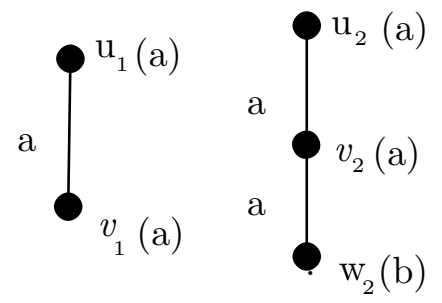

Here $G_{1}^{S} \cup G_{2}^{S}=(V, E, \sigma, \psi)$ where $V=V_{1} \cup V_{2} ; E=E_{1} \cup E_{2}$ such that, $\sigma\left(u_{1}\right)=\sigma_{1}\left(u_{1}\right)$ and $\sigma\left(v_{1}\right)=\sigma_{1}\left(v_{1}\right)\left(\because u_{1}, v_{1} \in V_{1}\right)$

$$
\sigma\left(u_{2}\right)=\sigma_{2}\left(u_{2}\right) ; \sigma\left(v_{2}\right)=\sigma_{2}\left(v_{2}\right) ; \sigma\left(w_{2}\right)=\sigma_{2}\left(w_{2}\right)\left(\because u_{2}, v_{2}, w_{2} \in V_{2}\right)
$$

Similarly $\psi\left(u_{1}, v_{1}\right)=\psi_{1}\left(u_{1}, v_{1}\right), \psi\left(u_{2}, v_{2}\right)=\psi_{2}\left(u_{2}, v_{2}\right)$, and $\psi\left(v_{2}, w_{2}\right)=\psi_{2}\left(v_{2}, w_{2}\right)$

Now $G_{1} \cup G_{2}=\left(V=V_{1} \cup V_{2}, E=E_{1} \cup E_{2}\right)$ where $V=\left\{u_{1}, v_{1}, u_{2}, v_{2}, w_{2}\right\}$ and $E=\left\{\left(u_{1}, v_{1}\right),\left(u_{2}, v_{2}\right),\left(v_{2}, w_{2}\right)\right\}$

Since the existance of an $S$-graph corresponding to its underlying graph is not unique, we define $\sigma_{3}: V \rightarrow S$ such that

$\sigma_{3}\left(u_{1}\right)=a ; \sigma_{3}\left(v_{1}\right)=b ; \sigma_{3}\left(u_{2}\right)=a ; \sigma_{3}\left(v_{2}\right)=b ; \sigma_{3}\left(w_{2}\right)=b ;$

and $\psi_{3}: E \rightarrow S$ such that

$$
\psi_{3}\left(u_{1}, v_{1}\right)=\min \left\{\sigma_{3}\left(u_{1}\right), \sigma_{3}\left(v_{1}\right)\right\}=\min \{a, b\}=a \quad(\because a \preceq b)
$$

Similarly $\psi_{3}\left(u_{2}, v_{2}\right)=a, \psi_{3}\left(v_{2}, w_{2}\right)=b$

$\Rightarrow \sigma_{3}(v) \neq \sigma(v)$ for every $v \in V=V_{1} \cup V_{2}$ 
$\psi_{3}\left(v_{i}, v_{j}\right) \neq \psi\left(v_{i}, v_{j}\right)$ for every $\left(v_{i}, v_{j}\right) \in E=E_{1} \cup E_{2}$.

Therefore $\sigma_{3} \neq \sigma$ and $\psi_{3} \neq \psi$. Then $\left(G_{1} \cup G_{2}\right)^{S}$ is

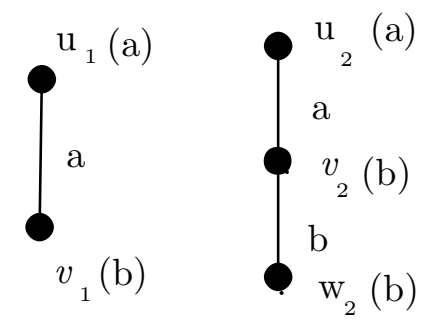

We observe that $G_{1}^{S} \cup G_{2}^{S} \neq\left(G_{1} \cup G_{2}\right)^{S}$ and the equality holds onlyif $\sigma=\sigma_{3}$.

Lemma 3.4. Union of two vertex regular $S$-graphs is a vertex regular $S$ graph if and only if their corresponding $S$-vertex sets are constant and assigns the same value in $S$.

Proof. Let $G_{1}^{S}=\left(V_{1}, E_{1}, \sigma_{1}, \psi_{1}\right)$ and $G_{2}^{S}=\left(V_{2}, E_{2}, \sigma_{2}, \psi_{2}\right)$ be two vertex regular $S$-graphs, corresponding to the underlying graphs $G_{1}=\left(V_{1}, E_{1}\right)$ and $G_{2}=\left(V_{2}, E_{2}\right)$ respectively.

Since $G_{1}^{S}$ and $G_{2}^{S}$ are vertex regular, $\sigma_{1}(v)=a$ for every $v \in V_{1}$, and some $a \in S$ and $\sigma_{2}(v)=b$ for every $v \in V_{2}$, and some $b \in S$,respectively. Hence $\sigma_{1}$ and $\sigma_{2}$ are constant. Let $G_{1}^{S} \cup G_{2}^{S}=(V, E, \sigma, \psi)$ be the union of given $S$-graphs and let it be vertex regular.

Therefore $\sigma(v)=c$ for every $v \in V$, and some $c \in S$.

Claim. $\sigma_{1}$ and $\sigma_{2}$ assigns the same value in $S$.

Let $v \in V_{1} \Rightarrow \sigma_{1}(v)=a$. Since $v \in V_{1} \subset V \Rightarrow \sigma(v)=c$. But $\sigma(v)=\sigma_{1}(v)$ $\Rightarrow c=a$.

Similarly, if $v \in V_{2} \Rightarrow \sigma_{2}(v)=b$. Since $v \in V_{2} \subset V \Rightarrow \sigma(v)=c$. But $\sigma(v)=\sigma_{2}(v) \Rightarrow c=b$. Therefore $a=b$.

Hence $\sigma_{1}$ and $\sigma_{2}$ are constant and assigns the same value in $S$.

Conversely, Let $\sigma_{1}$ and $\sigma_{2}$ be constant and assigns the same value in $S$. Let $a \in S$. and $\sigma_{1}(v)=a$ for all $v \in V_{1}, \sigma_{2}(v)=a$ for all $v \in V_{2}$.

Let $v \in V$ be arbitrary.Then

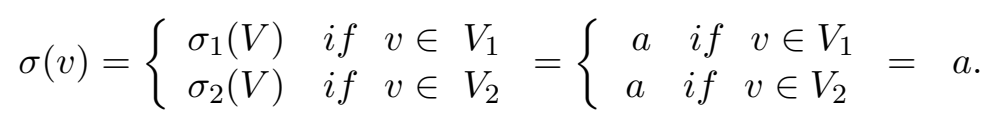

Hence $\sigma(v)=a, \forall v \in V$.

Therefore $G_{1}^{S} \cup G_{2}^{S}$ is a vertex regular $S$-graph. 
Corollary 3.5. Union of two vertex regular $S$-graphs is $S$-regular if and only if their corresponding $S$-vertex sets are constant and assigns the same value in $S$.

Lemma 3.6. Union of two edge regular $S$-graphs is an edge regular $S$ graph if and only if their corresponding $S$-edge sets are constant and assigns the same value in $S$.

Proof. Let $G_{1}^{S}=\left(V_{1}, E_{1}, \sigma_{1}, \psi_{1}\right)$ and $G_{2}^{S}=\left(V_{2}, E_{2}, \sigma_{2}, \psi_{2}\right)$ be two edge regular

$S$-graphs, corresponding to the underlying graphs $G_{1}=\left(V_{1}, E_{1}\right)$ and $G_{2}=\left(V_{2}, E_{2}\right)$ respectively.

Since $G_{1}^{S}$ and $G_{2}^{S}$ are edge regular, $\psi_{1}\left(v_{i}, v_{j}\right)=a$, for every $\left(v_{i}, v_{j}\right) \in E_{1}$, and some $a \in S$ and $\psi_{2}\left(v_{i}, v_{j}\right)=b$ for every $\left(v_{i}, v_{j}\right) \in E_{2}$ and some $b \in S$. then both $\psi_{1}$ and $\psi_{2}$ are constant. Let $G_{1}^{S} \cup G_{2}^{S}=(V, E, \sigma, \psi)$ be the union of the given $S$-graphs and let it be an edge regular $S$-graph.

Then $\psi\left(v_{i}, v_{j}\right)=c$ for every $\left(v_{i}, v_{j}\right) \in E$, and some $c \in S$.

Claim. $\psi_{1}$ and $\psi_{2}$ assigns the same value in $S$.

Let $\left(v_{i}, v_{j}\right) \in E_{1} \Rightarrow \psi_{1}\left(v_{i}, v_{j}\right)=a$. Since $\left(v_{i}, v_{j}\right) \in E_{1} \subset E \Rightarrow \psi\left(v_{i}, v_{j}\right)=c$.

But $\psi\left(v_{i}, v_{j}\right)=\psi_{1}\left(v_{i}, v_{j}\right)$. Hence $c=a$.

Similarly, if $\left(v_{i}, v_{j}\right) \in E_{2}$, we can prove that $c=b$. Therefore $a=b$. That is, $\psi_{1}$ and $\psi_{2}$ are constant and assigns the same value in $S$.

Conversely, assume that $\psi_{1}$ and $\psi_{2}$ are constant and assigns the same value in $S$. Let $a \in S . \psi_{1}\left(v_{i}, v_{j}\right)=a, \forall\left(v_{i}, v_{j}\right) \in E_{1} ; \psi_{2}\left(v_{i}, v_{j}\right)=a, \forall\left(v_{i}, v_{j}\right) \in E_{2}$.

Let $\left(v_{i}, v_{j}\right) \in E$ be arbitrary.Then

$$
\psi\left(v_{i}, v_{j}\right)=\left\{\begin{array}{ll}
\psi_{1}\left(v_{i}, v_{j}\right) & \text { if } \quad\left(v_{i}, v_{j}\right) \in E_{1} \\
\psi_{2}\left(v_{i}, v_{j}\right) & \text { if } \quad\left(v_{i}, v_{j}\right) \in E_{2}
\end{array}=\left\{\begin{array}{ll}
a & \text { if } \quad\left(v_{i}, v_{j}\right) \in E_{1} \\
a & \text { if } \quad\left(v_{i}, v_{j}\right) \in E_{2}
\end{array}=a\right.\right.
$$

That is $\psi\left(v_{i}, v_{j}\right)=a$ for all $\left(v_{i}, v_{j}\right) \in E$

Therefore $G_{1}^{S} \cup G_{2}^{S}$ is an edge regular $S$-graph.

Definition 3.7. Let $G_{1}^{S}=\left(V_{1}, E_{1}, \sigma_{1}, \psi_{1}\right)$ and $G_{2}^{S}=\left(V_{2}, E_{2}, \sigma_{2}, \psi_{2}\right)$ be two

$S$-graphs corresponding to the underlyings graphs $G_{1}=\left(V_{1}, E_{1}\right)$ and $G_{2}=$ $\left(V_{2}, E_{2}\right)$ with $V_{1} \cap V_{2}=\phi$ respectively. Then their sum, denoted by $G_{1}^{S}+G_{2}^{S}$, is defined as $G_{1}^{S}+G_{2}^{S}=(V, E, \sigma, \psi)$ where:

1. $V=V_{1} \cup V_{2} ; E=E_{1} \cup E_{2} \cup\left\{\left(v_{i}, v_{j}\right) \mid v_{i} \in V_{1}\right.$ and $\left.v_{j} \in V_{2}\right\}$ 
2. for $v \in V, \sigma(v)=\left\{\begin{array}{ll}\sigma_{1}(v) & \text { if } v \in V_{1} \\ \sigma_{2}(v) & \text { if } v \in V_{2}\end{array}\right.$.

3. for $\left(v_{i}, v_{j}\right) \in E$,

$$
\psi\left(v_{i}, v_{j}\right)=\left\{\begin{array}{cc}
\psi_{1}\left(v_{i}, v_{j}\right) & \text { if }\left(v_{i}, v_{j}\right) \in E_{1} \\
\psi_{2}\left(v_{i}, v_{j}\right) & \text { if }\left(v_{i}, v_{j}\right) \in E_{2} \\
\min \left\{\sigma_{1}\left(v_{i}\right), \sigma_{2}\left(v_{j}\right)\right\} & \text { if } v_{i} \in V_{1} \text { and } v_{j} \in V_{2}
\end{array}\right.
$$

Remark 3.8. Clearly sum of two $S$-graphs is also an $S$-graph.

Lemma 3.9. Sum of two vertex regular $S$-graphs is an vertex regular $S$-graph iff their corresponding $S$-vertex sets are constant and assigns the same value in $S$.

Proof. Let $G_{1}^{S}=\left(V_{1}, E_{1}, \sigma_{1}, \psi_{1}\right)$ and $G_{2}^{S}=\left(V_{2}, E_{2}, \sigma_{2}, \psi_{2}\right)$ be two vertex regular $S$-graphs, corresponding to the underlying graphs $G_{1}=\left(V_{1}, E_{1}\right)$ and $G_{2}=\left(V_{2}, E_{2}\right)$ respectively.

Since $G_{1}^{S}$ and $G_{2}^{S}$ are vertex regular,

$\sigma_{1}(v)=a$ for every $v \in V_{1}$, and some $a \in S$ then $\sigma_{1}$ is constant and $\sigma_{2}(v)=b$ for every $v \in V_{2}$, and some $b \in S$ then $\sigma_{2}$ is constant.

Let $G_{1}^{S}+G_{2}^{S}=(V, E, \sigma, \psi)$ be the sum of given $S$-graphs and let it be vertex regular $S$-graph.

Therefore $\sigma(v)=c$ for every $v \in V$, and some $c \in S$.

Claim. $\sigma_{1}$ and $\sigma_{2}$ assigns the same value in $S$.

Let $v \in V_{1} \Rightarrow \sigma_{1}(v)=a$.

Since $v \in V_{1} \subset V \Rightarrow \sigma(v)=c$.

But $\sigma(v)=\sigma_{1}(v) \Rightarrow c=a$.

Similarly, if $v \in V_{2}$ we can prove that $c=b$. Therefore $a=b$.

Hence $\sigma_{1}$ and $\sigma_{2}$ assigns the same value in $S$.

Conversely, assume that $\sigma_{1}$ and $\sigma_{2}$ are constant and assign the same value in $S$. Let $a \in S$ and $\sigma_{1}(v)=a$ for all $v \in V_{1}, \sigma_{2}(v)=a$, for all $v \in V_{2}$.

Let $v \in V$ be arbitrary. Then

$$
\sigma(v)=\left\{\begin{array}{ll}
\sigma_{1}(V) & \text { if } v \in V_{1} \\
\sigma_{2}(V) & \text { if } v \in V_{2}
\end{array}=\left\{\begin{array}{ll}
a & \text { if } v \in V_{1} \\
a & \text { if } v \in V_{2}
\end{array}=a .\right.\right.
$$

That is $\sigma(v)=a, \forall v \in V$ and $a \in S$.

Therefore $G_{1}^{S}+G_{2}^{S}$ is a vertex regular $S$-graph.

Corollary 3.10. Sum of two vertex regular $S$-graphs is $S$-regular if and only if their corresponding $S$-vertex sets are constant and assigns the same value in $S$. 
Remark 3.11. If $G_{1}^{S}$ and $G_{2}^{S}$ are edge regular $S$-graphs then their sum need not be, in general, an edge regular $S$-graph.

Proof. Let $(S=\{0, a, b\},+, \cdot)$ be a semiring with the following Cayley Tables:

\begin{tabular}{|c|c|c|c|}
\hline+ & 0 & $a$ & $b$ \\
\hline 0 & 0 & $a$ & $b$ \\
\hline$a$ & $a$ & $o$ & $b$ \\
\hline$b$ & $b$ & $b$ & $b$ \\
\hline
\end{tabular}

\begin{tabular}{|c|c|c|c|}
\hline$\cdot$ & 0 & $a$ & $b$ \\
\hline 0 & 0 & 0 & 0 \\
\hline$a$ & 0 & 0 & 0 \\
\hline$b$ & 0 & 0 & $b$ \\
\hline
\end{tabular}

Clearly $\preceq$ is a canonical pre-order in $S$, where

$$
0 \preceq 0,0 \preceq a, 0 \preceq b, a \preceq a, a \preceq 0, a \preceq b, b \preceq b .
$$

Let $G_{1}^{S}$ and $G_{2}^{S}$ be two edge regular but not vertex regular $S$-graphs given by :
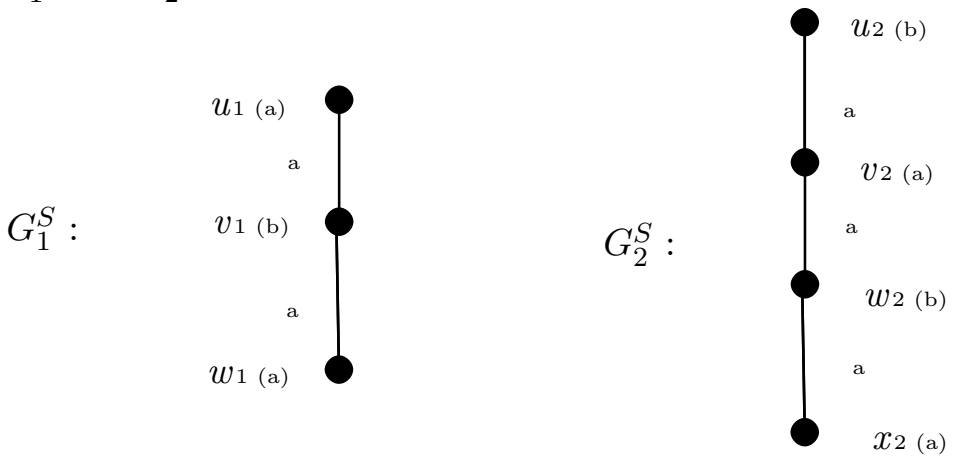

Here $G_{1}^{S}+G_{2}^{S}=(V, E, \sigma, \psi)$ where

$V=V_{1}+V_{2} ; E=E_{1} \cup E_{2} \cup\left\{\left(v_{i}, v_{j}\right) \mid v_{i} \in V_{1}\right.$ and $\left.v_{j} \in V_{2}\right\}$ such that,

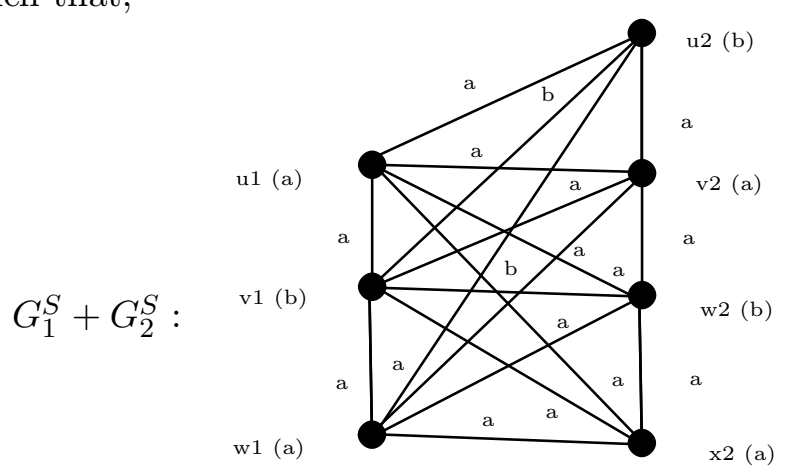

Here $\psi\left(v_{i}, v_{j}\right) \neq a$, for all $\left(v_{i}, v_{j}\right) \in E$.

Therefore $G_{1}^{S}+G_{2}^{S}$ is not an edge regular $S$-graph. 
Theorem 3.12. Sum of two edge regular $S$-graphs is an edge regular $S$-graph only if their corresponding $S$-vertex sets are constants and assigns the same value in $S$.

Proof. Let $G_{1}^{S}=\left(V_{1}, E_{1}, \sigma_{1}, \psi_{1}\right)$ and $G_{2}^{S}=\left(V_{2}, E_{2}, \sigma_{2}, \psi_{2}\right)$ be two edge regular $S$-graphs, corresponding to the underlying graphs $G_{1}=\left(V_{1}, E_{1}\right)$ and $G_{2}=\left(V_{2}, E_{2}\right)$ respectively.

Since $G_{1}^{S}$ and $G_{2}^{S}$ are edge regular.

$\psi_{1}\left(v_{i}, v_{j}\right)=a$ for every $\left(v_{i}, v_{j}\right) \in E_{1}$, and some $a \in S$.

$\psi_{2}\left(v_{i}, v_{j}\right)=b$ for every $\left(v_{i}, v_{j}\right) \in E_{2}$, and some $b \in S$.

Claim. $G_{1}^{S}+G_{2}^{S}$ is an edge regular $S$-graph only if their corresponding $S$-vertex sets assigns the same value in $S$.

Case 1. Suppose the $S$-vertex sets of $G_{1}^{S}$ and $G_{2}^{S}$ are constants and assigns the same value.

By corollary $3.10 G_{1}^{S}+G_{2}^{S}$ is a $S$-regular graph. Therefore it is an edge regular $S$-graph.

Case 2. Suppose $G_{1}^{S}$ and $G_{2}^{S}$ are edge regular and corresponding $S$-vertex sets are constant and $\sigma_{1}\left(v_{i}\right)=a$ forsome $a \in S$ and for every $v_{i} \in V_{1}$; and $\sigma_{2}\left(v_{j}\right)=b$ forsome $b \in S$ and for every $v_{j} \in V_{2}$ such that $a \neq b$. Then for $\left(v_{i}, v_{j}\right) \in E$,

$$
\begin{aligned}
& \psi\left(v_{i}, v_{j}\right)=\left\{\begin{array}{cc}
\psi_{1}\left(v_{i}, v_{j}\right) & \text { if }\left(v_{i}, v_{j}\right) \in E_{1} \\
\psi_{2}\left(v_{i}, v_{j}\right) & \text { if }\left(v_{i}, v_{j}\right) \in E_{2} \\
\min \left\{\sigma_{1}\left(v_{i}\right), \sigma_{2}\left(v_{j}\right)\right\} & \text { if } v_{i} \in V_{1}, v_{j} \in V_{2}
\end{array}\right. \\
& =\left\{\begin{array}{cc}
a & \text { if }\left(v_{i}, v_{j}\right) \in E_{1} \\
b & \text { if }\left(v_{i}, v_{j}\right) \in E_{2} \\
\min \{a, b\} & \text { if } v_{i} \in V_{1}, v_{j} \in V_{2},
\end{array}\right.
\end{aligned}
$$

Since the edges in $E_{1}$ and $E_{2}$ assumes different values, we have $\psi\left(v_{i}, v_{j}\right)$ is not a constant for every $\left(v_{i}, v_{j}\right) \in E$.

Therefore $G_{1}^{S}+G_{2}^{S}$ is not an edge regular $S$-graph.

Case 3. Suppose $G_{1}^{S}$ and $G_{2}^{S}$ are edge regular and corresponding $S$-vertex sets are not constant. Then there exists some $v_{k} \in V_{1}$ such that $\sigma_{1}\left(v_{k}\right)=c$ for some $c \in S$, and $c \neq a$.

Similarly there exists some $v_{l} \in V_{2}$ such that $\sigma_{2}\left(v_{l}\right)=d$ for some $d \in S$, and $d \neq b$. Clearly $\left(v_{l}, v_{k}\right)$ or $\left(v_{k}, v_{l}\right) \in E$. Then

$$
\psi\left(v_{k}, v_{l}\right)=\min \{c, d\}=\left\{\begin{array}{cc}
c & \text { if } \sigma_{1}\left(v_{k}\right) \preceq \sigma_{2}\left(v_{l}\right) \\
d & \text { if } \sigma_{2}\left(v_{l}\right) \preceq \sigma_{1}\left(v_{k}\right) \\
0 & \text { otherwise }
\end{array}\right.
$$


In any case, $\psi\left(v_{i}, v_{j}\right)$ is not a constant for all $\left(v_{i}, v_{j}\right) \in E$.

Thus the sum of two edge regular $S$-graphs is an edge regular $S$-graph only if their corresponding $S$-vertex sets are constants and assigns the same value in $S$.

Theorem 3.13. Union(Sum) of two $(a, k)$-regular $S$-graphs is $S$-regular.

Proof. Since every $(a, k)$-regular $S$-graph is an $S$-regular, and union(sum) of two $S$-regular is an $S$-regular, we have union(sum) of two $(a, k)$-regular graph is $S$-regular.

\section{Conclusion}

Unlike the crisp graph theory, in $S$-graphs, we define the union and sum of two $S$-graphs by considering the $S$-values of both vertices and edges. In our future work, we would like to extend the study of $S$-graphs subject to other operations such as cartesian products and composition of $S$-graphs.

\section{References}

[1] J.A. Bondy, U.S.R. Murty, Graph Theory with Applications, North Holland, New York (1982).

[2] Golan S. Jonathan, Semirings and Their Applications, Kluwer Academic Publishers, London.

[3] S. Jeyalakshmi, M. Rajkumar, M. Chandramouleeswaran, Regularity on S-Graphs, Submitted.

[4] M. Rajkumar, S. Jeyalakshmi, M. Chandramouleeswaran, Semiring-valued graphs, International Journal of Math. Sci. and Engg. Appls., 9, No. 3 (2015), 141-152.

[5] H.S. Vandiver, Note on a simple type of algebra in which the cancellation law of addition does not hold, Bull. Amer. Math. Soc., 40 (1934), 916-920. 